\title{
Delay in Diagnostic Workup and Treatment of Esophageal Cancer
}

\author{
Brechtje A. Grotenhuis • Pieter van Hagen • \\ Bas P. L. Wijnhoven • Manon C. W. Spaander • \\ Hugo W. Tilanus • Jan J. B. van Lanschot
}

Received: 15 September 2009 / Accepted: 9 November 2009 /Published online: 10 December 2009

(C) 2009 The Author(s). This article is published with open access at Springerlink.com

\begin{abstract}
Introduction Esophageal cancer should preferably be detected and treated at an early stage, but this may be prohibited by late onset of symptoms and delays in referral, diagnostic workup, and treatment. The aim of this study was to investigate the impact of these delays on outcome in patients with esophageal cancer.

Methods For 491 patients undergoing esophagectomy for cancer between 1991 and 2007, patients' short- and long-term outcome were analyzed according to different time intervals between onset of symptoms, diagnosis, and surgical treatment.

Results Length of prehospital delay (from onset of symptoms until endoscopic diagnosis) did not affect patient's short- or long-term outcome. A shorter hospital delay between establishing the diagnosis of esophageal cancer on endoscopy and surgery was associated with lower overall morbidity and in-hospital mortality. Patients of ASA classes I and II experienced a shorter hospital delay than patients of ASA classes III and IV. Length of hospital delay between endoscopic diagnosis and surgery did not affect pathological tumor-node-metastasis stage or R0-resection rate. Longer hospital delay did not result in worse survival: Overall survival after esophagectomy for cancer was not significantly different between patients with hospital delay $<5,5-8$, or $>8$ weeks $(24.7 \%, 21.7 \%$, and $32.3 \%$, respectively; $p=0.12)$.

Conclusion A longer hospital delay (between endoscopic diagnosis and surgery) resulted in worse patient's short-term outcome (higher overall morbidity and mortality rates) but not in a worse long-term outcome (overall survival). This may be explained by a more time-consuming diagnostic workup in patients with a poorer physical status and not by tumor progression.
\end{abstract}

Keywords Esophageal cancer· Diagnostic workup · Delay · Waiting list · Survival

B. A. Grotenhuis · P. van Hagen · B. P. L. Wijnhoven •

H. W. Tilanus · J. J. B. van Lanschot

Department of Surgery, Erasmus Medical Center,

Erasmus University,

Rotterdam, The Netherlands

M. C. W. Spaander

Department of Gastroenterology, Erasmus Medical Center,

Erasmus University,

Rotterdam, The Netherlands

B. A. Grotenhuis $(\bowtie)$

Erasmus Medical Center, Department of Surgery,

PO Box 2040, 3000 CA Rotterdam, The Netherlands

e-mail: b.grotenhuis@erasmusmc.nl

\section{Introduction}

The 5-year survival rate for esophageal cancer patients after esophagectomy with curative intent has improved up to $40 \%{ }^{1-3}$ As further improvement in survival from a single modality approach, such as surgery, is unlikely, considerable interest has grown in other strategies that may improve patients' survival (neoadjuvant chemo- and/or radiotherapy in particular). In many types of cancer, the prognosis of patients with small, localized tumors is better than with locally advanced or metastatic disease. Similar to other malignancies such as colorectal and breast cancer, the outcome of esophageal cancer is related to the pathological tumor-node-metastasis (pTNM) stage of the disease. ${ }^{2,4,5}$ Therefore, detection and treatment of esophageal cancer at an early stage could also improve long-term survival. 
Early detection of esophageal cancer may be prohibited not only by the late onset of symptoms but also by delays in referral to an appropriate specialist, establishment of the diagnosis, further diagnostic workup, and start of treatment. However, the impact of these delays on both short- and long-term outcome for patients undergoing esophagectomy for cancer is unclear.

In patients with breast cancer, delays of 3-6 months between the onset of symptoms and start of treatment are associated with lower survival, caused by a more advanced tumor stage. ${ }^{6}$ In two systematic reviews, no association was found between diagnostic and therapeutic delay and survival in colorectal cancer patients ${ }^{7}$ nor between these delays and disease stage. ${ }^{8}$ A few studies have investigated the impact of delays in diagnosis and treatment of esophageal cancer. Drawbacks of these studies are small numbers of patients included, ${ }^{9}$ analyses that do not cover the complete track between onset of symptoms and surgical treatment, ${ }^{10,11}$ combined patient groups with gastric and esophageal carcinoma, ${ }^{12,13}$ and studies lacking survival analyses. $^{9,11-13}$

We hypothesized that longer delays between onset of symptoms, endoscopic diagnosis, and surgical treatment are associated with a worse short-term outcome (morbidity, reoperation rate, and in-hospital mortality), worse tumor stage, and hence, worse long-term outcome (overall survival) following potentially curative esophagectomy in patients with esophageal cancer.

\section{Patients and Methods}

The Erasmus Medical Center in Rotterdam is a tertiary referral center for patients with esophageal cancer in The Netherlands. Most patients are referred to the Erasmus MC outpatient clinic for (surgical) treatment after the diagnosis of esophageal cancer has been established in a referring hospital (group A). The minority of patients is directly referred by the general practitioner (GP) to the Erasmus $\mathrm{MC}$ for clinical investigations of symptoms suggestive of cancer (group B). In all patients (groups A and B) upper gastrointestinal endoscopy with biopsy is (re)done in the Erasmus MC to confirm the diagnosis of esophageal cancer and to determine the exact location of the tumor. Staging is performed routinely with endoscopic ultrasonography, CT scanning of thorax and abdomen, and external ultrasound of the neck. Every patient is discussed in a weekly multidisciplinary oncology meeting in which a definitive treatment plan is designed. If eligible for surgery, patients are put on the waiting list for surgery. On the same day, the patient is referred to the Department of Anesthesiology for preoperative counseling. If needed, additional cardiac and/or pulmonary function tests are scheduled.
Between January 1991 and December 2007, 791 patients underwent esophagectomy for cancer of the esophagus or gastroesophageal junction in the Erasmus MC. To obtain a homogeneous cohort of patients in terms of treatment and to circumvent possible stage migration following chemoand/or radiotherapy, patients receiving (neo)adjuvant therapy were excluded from this analysis. In our hospital, patients received neoadjuvant chemo(radio)therapy in the context of randomized controlled trials. ${ }^{14,15}$ Induction chemo- and/or radiotherapy was given in patients with either a cT4 tumor without distant metastases or in patients with gross involvement of celiac trunk lymph nodes (M1a), who were not considered eligible for primary surgical therapy. There were 214 patients who were excluded because of chemo- and/or radiotherapy prior to surgery. In 44 patients, the hospital delay from endoscopic diagnosis to surgery could not be calculated, as the date of their first upper gastrointestinal endoscopy performed in the referring hospital was unknown. Another 42 patients were excluded, as they participated in a Barrett's esophagus surveillance program. Over recent years, multiple attempts for endoscopic treatment of early lesions delayed referral to the Department of Surgery in such way that this group was not representative for patients treated for (more advanced) esophageal cancer. Finally, data of 491 patients were analyzed in the present study. The vast majority of these patients underwent a transhiatal esophagectomy with locoregional lymphadenectomy only $(N=477)$. In 14 patients, a transthoracic resection with extended lymphadenectomy was performed. The applied surgical techniques have been described previously. ${ }^{3,16}$ Tumors were assigned pTNM stages according to the Union Internationale Contre le Cancer 2002 system. $^{17}$

Data on patients' demographics, diagnostic tests, surgery, postoperative morbidity, in-hospital mortality, and survival have been collected prospectively and stored in a database by a data manager. From this database, the following time points were defined:

- Date of upper gastrointestinal endoscopy in the referring hospital, on which the diagnosis of esophageal cancer had been established by histology from biopsies (only applicable for group A)

- Date of first visit at the Erasmus MC outpatient clinic: Department of Surgery, Gastroenterology, or Medical Oncology

- Date of upper gastrointestinal endoscopy in the Erasmus MC, on which the diagnosis of esophageal cancer had been established by histology from biopsies

- Date of the multidisciplinary oncology meeting, after which the patient had been put on the operative waiting list if eligible for surgery

- Date of surgery. 
To summarize all different time points that have been marked in the process between onset of symptoms and surgery, we divided this time span into two major time intervals that have been analyzed separately: pre-hospital and hospital delay (see Fig. 1). Subsequently, data were analyzed in three different ways:

- Impact of prehospital delay: time from onset of symptoms until diagnosis on first endoscopy (either in the referring hospital for group A or in Erasmus MC for group B)

- Impact of hospital-delay: time from diagnosis on patient's first endoscopy undertaken until surgery

- Impact of specific time intervals between diagnosis on first endoscopy and surgery. In order to examine the hospital-delay in more detail, the effect of specific time intervals between diagnosis in the referring hospital, first visit at the outpatient clinic in Erasmus MC, diagnosis on endoscopy in Erasmus MC, multidisciplinary oncology meeting, and surgery on short- and long-term outcome were analyzed. For this purpose, only data from patients in group A were used.

\section{Statistics}

Follow-up was recorded until December 2008 or until death if earlier and was complete for all patients. Statistical analysis for non-parametric data was used. Grouped data were compared using the chi-square, Mann-Whitney $U$, or Kruskall-Wallis $H$ test. Patients who died due to complications following esophagectomy (in-hospital mortality) were not excluded from survival analysis. Overall survival was calculated from the date of operation until the date of last follow-up or death according to the Kaplan-Meier method. Disease-free survival was assessed from the date of operation until the date of disease recurrence in case of locoregional recurrence or distant metastases. Univariate analyses were performed with the log-rank test to identify prognostic variables associated with overall survival after esophagectomy. Data analyses were carried out with SPSS version 15.0 (SPSS, Chicago, IL, USA).

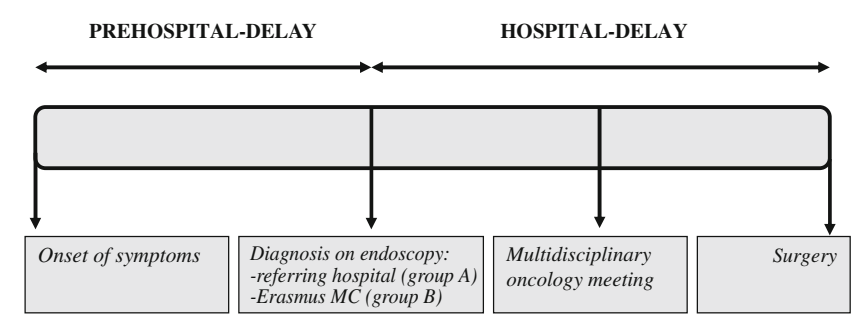

Figure 1 Analysis of prehospital and hospital delays encountered by patients who underwent surgical resection for esophageal cancer in Erasmus MC.
Table 1 Clinicopathological Characteristics of 491 Patients who Underwent Surgical Resection for Esophageal Cancer and Who Were Included in the Present Study

\begin{tabular}{lc}
\hline Age (in years) $^{\text {a }}$ & $65(28-89)$ \\
Gender & \\
Male & $399(81.3 \%)$ \\
Female & $92(18.7 \%)$ \\
ASA classification & \\
I & $77(15.7 \%)$ \\
II & $316(64.4 \%)$ \\
III & $96(19.6 \%)$ \\
IV & $2(0.4 \%)$ \\
Tumor location & $8(1.6 \%)$ \\
Proximal esophagus & $27(5.5 \%)$ \\
Mid esophagus & $196(39.9 \%)$ \\
Distal esophagus & $260(53.0 \%)$ \\
Gastroesophageal junction & \\
Histology & $73(14.9 \%)$ \\
Squamous cell carcinoma & $418(85.1 \%)$ \\
Adenocarcinoma &
\end{tabular}

ASA classification American Society of Anesthesiologists classification

${ }^{\text {a }}$ Age is given as median (range)

\section{Results}

Patients' characteristics are shown in Table 1. Three hundred sixty-five patients (74.3\%), in whom the diagnosis esophageal cancer was established in another hospital, were referred to the Erasmus $\mathrm{MC}$ for further staging and treatment (group A). One hundred twenty-six patients (25.7\%) were referred directly to the Erasmus MC by the general practitioner for investigation of symptoms suggestive of esophageal cancer (group B). Patients' first visit to the Erasmus MC was at the Department of Surgery $(N=$ $338,68.8 \%)$, Department of Gastroenterology $(N=147$, $29.9 \%)$, or Department of Medical Oncology $(N=6,1.3 \%)$.

Impact of Prehospital Delay: Time from Onset of Symptoms Until First Endoscopy

The majority of patients underwent endoscopy for investigation of obstructive symptoms suggestive of cancer like dysphagia, odynophagia, and weight loss $(N=462,94.1 \%)$. Other indications for endoscopy encompassed investigation of hematemesis $(N=12,2.4 \%)$, anemia $(N=9,1.8 \%)$, or melena $(N=8,1.6 \%)$. Prehospital delay (from onset of symptoms until first endoscopy) lasted a median time period of 3.0 months (range, 0-36 months). Patient's short-term (morbidity, reoperation rate, and in-hospital mortality) and long-term outcome (overall 5-year survival) after esophagectomy were comparable for patients who experienced 
Table 2 Impact of Prehospital Delay from Onset of Symptoms to First Endoscopy on Shortand Long-Term Outcome After Esophagectomy; Comparison of Prehospital Delay $\leq 3$ Months $(N=308)$ Versus $>3$ Months $(N=183)$

\begin{tabular}{llll}
\hline & $\begin{array}{l}\text { Prehospital delay } \leq 3 \text { months, } \\
N=308\end{array}$ & $\begin{array}{l}\text { Prehospital delay }>3 \text { months, } \\
N=183\end{array}$ & $p$ value \\
\hline Morbidity & $199(64.6 \%)$ & $104(56.8 \%)$ & 0.09 \\
Reoperation & $34(11.0 \%)$ & $16(8.7 \%)$ & 0.42 \\
In-hospital mortality & $18(5.8 \%)$ & $9(4.9 \%)$ & 0.66 \\
Overall 5-year survival & $24.0 \%$ & $29.3 \%$ & 0.10 \\
\hline
\end{tabular}

symptoms for a period of 3 months or less versus more than 3 months until endoscopy was performed (Table 2).

Impact of Hospital Delay: Time from Endoscopic Diagnosis Until Surgery

The hospital delay from establishing the diagnosis of esophageal cancer on endoscopy (either in the referring hospital for group A or in Erasmus MC for group B) until surgery was 49 days (given as median, range of 5175 days). This delay encompassed a median time period of 28 days (range, 0-147 days) from diagnosis on patient's first endoscopy until the multidisciplinary oncology meeting (staging delay), and a median time period of 15 days from this meeting until surgery (operative waiting list, range of 1-67 days). Median hospital delay between diagnosis and surgery increased during the study period (1991-2007): 3.9 weeks in 1991 toward 10.9 weeks in 2007 (Fig. 2). This increase in hospital delay should rather be ascribed to the 3.4 times increase in length of the operative waiting list (1.6 weeks in 1991 towards 5.6 weeks in 2007) than to the 1.5 times increase in staging delay (3.3 weeks in 1991 towards 4.9 weeks in 2007).

A shorter hospital delay between establishing the diagnosis of esophageal cancer on patient's first endoscopy and surgery was associated with significantly lower overall morbidity and mortality (Table 3). These associations

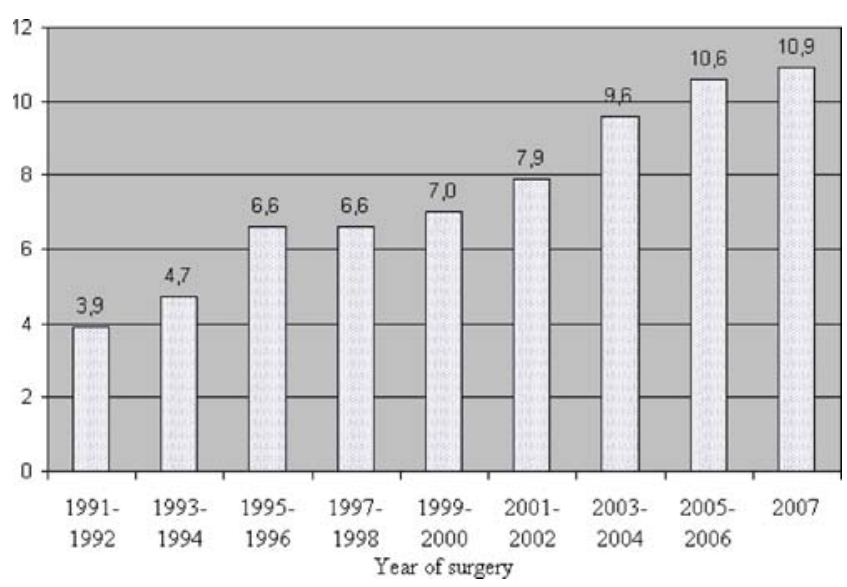

Figure 2 Median hospital delay (in weeks) between endoscopic diagnosis and surgery increased during the study period (1991-2007): 3.9 weeks in 1991 toward 10.9 weeks in 2007. appeared to be linear: morbidity $(p=0.001)$ and in-hospital mortality $(p=0.01)$ increased with longer hospital delay. Patients of ASA classes I and II experienced a shorter hospital delay than patients of ASA classes III and IV (hospital delay $<5$ weeks, 28.8\%; 5-8 weeks, 36.9\%; and $>8$ weeks, $34.4 \%$ versus $<5$ weeks, $15.3 \%$; 5-8 weeks, $41.8 \%$; and $>8$ weeks, $42.9 \%$, respectively; $p=0.02$ ). Length of hospital delay did not affect pTNM stage or R0resection rate (Table 3 ).

Longer hospital delay did not result in worse survival (Fig. 3): Overall 5-year survival was $24.7 \%$ in patients with a hospital delay less than 5 weeks, $21.7 \%$ in patients with a hospital delay between 5 and 8 weeks and $32.3 \%$ in patients in whom the hospital delay was more than 8 weeks. Although overall survival appeared to be longer in patients with a longer hospital delay, this difference was not statistically significant $(p=0.12)$. Parameters found to be associated with overall survival in univariate analyses are shown in Table 4: age younger than 65 years, early pT stage (pT1 or pT2), no lymph node involvement (pN0), absence of distant metastatic disease (pM0), good differentiation grade of the tumor, R0 resection, and lymph node ratio smaller than 0.24 were favorable of improved overall survival. Survival analysis with regard to 5-year diseasefree survival paralleled the overall 5-year survival curves $(27.0 \%, 27.7 \%$, and $38.3 \%$, respectively; $p=0.09)$.

\section{Impact of Specific Time Intervals Between Endoscopic Diagnosis and Surgery (Group A)}

The median hospital delay was 53 days (range, 5175 days) for patients in group $\mathrm{A}$ in whom the diagnosis esophageal cancer had been established in another hospital and who were referred to the Erasmus MC for surgical treatment $(N=365)$. The breakdown of this delay is shown in Table 5, according to the different time intervals between diagnosis in the referring hospital, first visit to the outpatient clinic in Erasmus MC, diagnosis on endoscopy in Erasmus MC, multidisciplinary oncology meeting, and surgery.

When analyzing the impact of the separate time intervals, it appeared that the delay between the multidisciplinary oncology meeting and surgery (median, 15 days; reflecting the length of the operative waiting list) was the only time interval that influenced short-term outcome post- 
Table 3 Impact of the Hospital Delay from Diagnosis on Patient's First Endoscopy Until Surgery: Hospital Delay $<5$ Weeks $(N=128), 5-8$ Weeks $(N=186)$, and $>8$ Weeks $(N=177)$

\begin{tabular}{|c|c|c|c|c|}
\hline & Delay $<5$ weeks, $N=128$ & Delay 5-8weeks, $N=186$ & Delay $>8$ weeks, $N=177$ & $p$ value \\
\hline Morbidity & $62(48.4 \%)$ & $122(65.6 \%)$ & $119(67.2 \%)$ & $<0.01$ \\
\hline In-hospital mortality & $2(1.6 \%)$ & $10(5.4 \%)$ & $15(8.5 \%)$ & 0.03 \\
\hline Reoperation & $7(5.5 \%)$ & $20(10.8 \%)$ & $23(13.0 \%)$ & 0.10 \\
\hline \multicolumn{5}{|l|}{ pT stage } \\
\hline pT1-pT2 & $30(23.4 \%)$ & $57(30.6 \%)$ & $54(30.5 \%)$ & \multirow[t]{2}{*}{0.31} \\
\hline pT3-pT4 & $98(76.6 \%)$ & $129(69.4 \%)$ & $123(69.5 \%)$ & \\
\hline \multicolumn{5}{|l|}{$\mathrm{pN}$ stage } \\
\hline pN0 & $42(32.8 \%)$ & $66(35.5 \%)$ & $62(35.0 \%)$ & \multirow[t]{2}{*}{0.88} \\
\hline $\mathrm{pN} 1$ & $86(67.2 \%)$ & $120(64.5 \%)$ & $115(65.0 \%)$ & \\
\hline \multicolumn{5}{|l|}{ pM stage } \\
\hline $\mathrm{pM} 0$ & $103(80.5 \%)$ & $150(80.6 \%)$ & $131(74.0 \%)$ & \multirow[t]{2}{*}{0.24} \\
\hline pM1a-M1b & $25(19.5 \%)$ & $36(19.4 \%)$ & $46(26.0 \%)$ & \\
\hline \multicolumn{5}{|l|}{ Radicality of resection } \\
\hline R0 & $86(67.2 \%)$ & $124(66.7 \%)$ & $130(73.4 \%)$ & \multirow[t]{2}{*}{0.32} \\
\hline $\mathrm{R} 1-\mathrm{R} 2$ & $42(32.8 \%)$ & $62(33.3 \%)$ & $47(26.6 \%)$ & \\
\hline
\end{tabular}

esophagectomy. Although in-hospital mortality was comparable between patients who had been on the waiting list for 15 days or shorter versus patients who were waiting for more than 15 days $(p=0.14)$, length of the operative waiting list did influence morbidity ( $55.7 \%$ versus $67.1 \%, p=0.03$ ), and a trend towards an increased reoperation rate could be noted $(7.8 \%$ versus $13.9 \%, p=0.06)$. However, in contrast with the hospital delay between endoscopic diagnosis and surgery, none of the separate time intervals affected longterm survival.

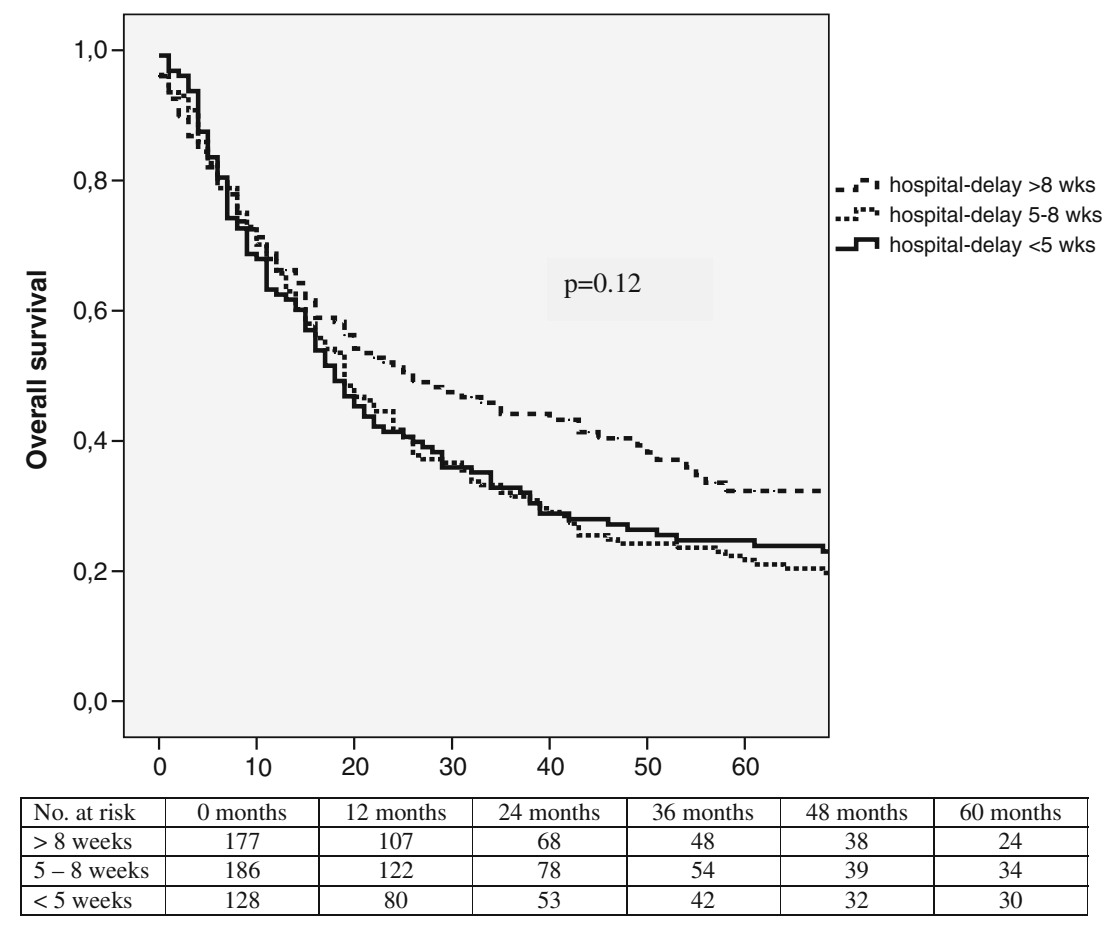

Figure 3 Overall 5-year survival for esophageal cancer patients appeared longer for patients with a hospital delay between diagnosis on first endoscopy and surgery $>8$ weeks $(N=177)$ versus patients with a hospital delay $<5$ weeks $(N=128)$ or $5-8$ weeks $(N=186)$, although this difference did not reach statistical significance $(p=0.12)$. 
Table 4 Univariate Analyses of Potential Prognostic Variables Associated with Overall Survival After Esophagectomy for Cancer $(N=491)$

\begin{tabular}{|c|c|c|}
\hline Variable & Five-year survival (\%) & $p$ value \\
\hline \multicolumn{3}{|l|}{ Age } \\
\hline$\leq 65$ years & 30.2 & \multirow[t]{2}{*}{0.001} \\
\hline$>65$ years & 21.4 & \\
\hline \multicolumn{3}{|l|}{ Sex } \\
\hline Male & 25.4 & \multirow[t]{2}{*}{0.84} \\
\hline Female & 28.5 & \\
\hline \multicolumn{3}{|l|}{ ASA classification } \\
\hline $\mathrm{I}-\mathrm{II}$ & 27.0 & \multirow[t]{2}{*}{0.12} \\
\hline III-IV & 22.2 & \\
\hline \multicolumn{3}{|l|}{ pT stage } \\
\hline $\mathrm{pT} 1-\mathrm{T} 2$ & 53.3 & \multirow[t]{2}{*}{$<0.001$} \\
\hline pT3-T4 & 15.0 & \\
\hline \multicolumn{3}{|l|}{$\mathrm{pN}$ stage } \\
\hline $\mathrm{pN} 0$ & 50.3 & \multirow[t]{2}{*}{$<0.001$} \\
\hline $\mathrm{pN} 1$ & 12.2 & \\
\hline \multicolumn{3}{|l|}{ pM stage } \\
\hline pM0 & 39.8 & \multirow[t]{2}{*}{$<0.001$} \\
\hline pM1a-M1b & 9.5 & \\
\hline \multicolumn{3}{|l|}{ Histology } \\
\hline Squamous cell carcinoma & 27.1 & \multirow[t]{2}{*}{0.98} \\
\hline Adenocarcinoma & 25.8 & \\
\hline \multicolumn{3}{|l|}{ Differentiation grade of tumor } \\
\hline Good & 69.1 & \multirow{3}{*}{$<0.001$} \\
\hline Moderate & 29.5 & \\
\hline Poor & 16.0 & \\
\hline \multicolumn{3}{|l|}{ Radicality of resection } \\
\hline $\mathrm{R} 0$ & 35.5 & \multirow[t]{2}{*}{$<0.001$} \\
\hline $\mathrm{R} 1-\mathrm{R} 2$ & 5.5 & \\
\hline \multicolumn{3}{|l|}{ Lymph node ratio } \\
\hline$\leq 0.24$ & 36.0 & \multirow[t]{2}{*}{$<0.001$} \\
\hline$>0.24$ & 12.0 & \\
\hline \multicolumn{3}{|l|}{ Referral } \\
\hline By another hospital (group A) & 25.9 & \multirow[t]{2}{*}{0.65} \\
\hline By GP (group B) & 26.2 & \\
\hline \multicolumn{3}{|l|}{ Prehospital delay } \\
\hline$\leq 3$ months & 24.0 & \multirow[t]{2}{*}{0.10} \\
\hline$>3$ months & 29.3 & \\
\hline \multicolumn{3}{|l|}{ Hospital delay } \\
\hline$<5$ weeks & 24.7 & \multirow{3}{*}{0.12} \\
\hline 5-8 weeks & 21.7 & \\
\hline$>8$ weeks & 32.3 & \\
\hline
\end{tabular}

ASA classification American Society of Anesthesiologists classification, GP general practitioner

\section{Discussion}

When initiating the current study, we hypothesized that longer delays between onset of symptoms, diagnosis, and surgical treatment are associated with worse short-term outcome (in terms of morbidity, reoperation rate, and mortality) and worse long-term outcome (overall survival) following esophagectomy for cancer. In the present series, it appeared that length of prehospital delay (from onset of symptoms until endoscopic diagnosis) did not influence patient's short-term outcome or overall 5-year survival. Onset of symptoms is a subjective measurement, and it may be that patients are not able to recall the exact moment that they first experienced discomfort. Furthermore, although little information is known about the tumor doubling time of esophageal cancer, the period of time in which a patient is symptomatic may be relatively short when compared to the total period between the first presence of malignant cells in the esophagus and the diagnosis of esophageal cancer. Unfortunately, we did not have information on delays caused by the GP (i.e., time between onset of symptoms and referral for endoscopy). Nevertheless, we do want to emphasize the importance of both patient and primary care education that will result in earlier notification of alarming symptoms such as dysphagia and weight loss.

A longer hospital delay from endoscopic diagnosis until surgery was associated with higher overall morbidity and mortality. This could be explained by a more thorough and time-consuming diagnostic workup in patients with a poorer physical status. Indeed, in the present study, patients of ASA classes I and II experienced a shorter hospital delay than patients of ASA classes III and IV. Alternatively, a longer delay prior to surgery may also have caused a worse physical status in esophageal cancer patients by means of malnutrition. However, this remains speculative, as our database did not provide detailed information with regard to patients' preoperative nutritional status (e.g., nutritional risk indices). When analyzing the impact of the separate time intervals between patient's first endoscopy and surgery, it appeared that the length of the operative waiting list was the time interval that influenced short-term outcome following esophagectomy the most. From the literature, it

Table 5 Delays Encountered by Esophageal Cancer Patients who have been Referred from an Other Hospital to the Erasmus MC for Surgical Treatment (group A, $N=365$ )

Diagnosis on endoscopy elsewhere $\rightarrow$ first visit outpatient clinic Erasmus MC

First visit outpatient clinic Erasmus $\mathrm{MC} \rightarrow$ diagnosis on endoscopy Erasmus MC

Diagnosis on endoscopy Erasmus $\mathrm{MC} \rightarrow$ multidisciplinary oncology meeting

Multidisciplinary oncology meeting $\rightarrow$ surgery

17 days (1-138)

Total hospital delay

Diagnosis on endoscopy elsewhere $\rightarrow$ surgery

6 days $(0-36)$

7 days (0-95)

15 days (1-67)

53 days (5-175)

Lengths of delays are given as a median values with the corresponding range in brackets 
is also known that the quality of life in newly diagnosed esophageal cancer patients who are waiting for surgery is seriously impaired. ${ }^{18}$ Hence, it should be aimed for to keep this time interval to a minimum.

Our second hypothesis was that patients with longer delays would generally present with more advanced disease and that this relation between delay and stage would result in a poorer survival. However, pTNM stages were comparable in patients with a hospital delay $<5,5-8$, or $>8$ weeks between endoscopy and surgery. Surprisingly, it appeared that overall survival was improved in patients with a longer hospital delay, although this difference was not statistically significant. This is in line with the results of Kötz et al. ${ }^{10}$ who showed that a longer delay between diagnosis and surgical resection was associated with improved survival in esophageal cancer patients. However, the delay between diagnosis and surgery was not an independent prognostic variable on multivariate analysis in their study. Kötz et al. ${ }^{10}$ noted that patients with a longer delay had a higher rate of complete tumor resection, suggesting that they were more appropriately selected for surgical treatment. In our series, we could not find evidence that patients were selected more appropriately, as both pTNM stage and R0-resection rate did not differ between patients with a shorter or longer hospital delay. However, hospital delay substantially increased especially over the last few years in our hospital (Fig. 2). This can probably explain the counter-intuitive correlation between longer hospital delay and improved long-term survival, which is rather reflecting state-of-the-art staging modalities, refined surgical techniques, and improved intensive care that have been introduced over the past years. Theoretically, it could also be possible that, in our hospital, patients did not undergo surgery anymore after a longer hospital delay in case the tumor progressed to a stage that was considered irresectable. However, in our patient group, the increased hospital delay can rather be ascribed to an increase in length of the operative waiting list than to an increased staging delay. As the decision on whether to operate or not has been made during the multidisciplinary oncology meeting, it is unlikely that a longer hospital delay led to a dropout of patients with irresectable tumors and, hence, a more selected patient group that underwent esophagectomy.

It is evident that efforts are taken to minimize delays experienced by patients with esophageal cancer between onset of symptoms, diagnosis, and surgical treatment. The National Health Service cancer plan was implemented in 2000 in the UK, indicating that all patients with relevant symptoms and suspected cancer should be able to see a specialist within 2 weeks of their GP referral. The introduction of these guidelines was associated with reductions in times to first outpatient visit, endoscopy, and diagnosis in patients with upper gastrointestinal cancer (esophageal or gastric). ${ }^{19,20}$ However, the effectiveness of the NHS cancer plan is uncertain, as it can be questioned whether the slightly improved survival rates after 2000 can be ascribed to this plan. ${ }^{21}$

In our hospital, we recently introduced a new schedule of diagnostic services for patients with suspected esophageal cancer. It is attempted to see patients at the outpatient clinic of the Department of Surgery or Department of Gastroenterology within 1 week after referral. Furthermore, patients are offered all imaging modalities in 1 week, including upper gastrointestinal endoscopy, endoscopic ultrasonography, CT scanning of thorax and abdomen, and external ultrasound of the neck. The aim of this schedule is to minimize the delay between referral to our hospital and establishment of a definitive treatment plan for each individual patient.

In conclusion, length of prehospital delay (from onset of symptoms until diagnosis) did not affect patient's short- or long-term outcome. A longer hospital delay (between endoscopic diagnosis and surgery) resulted in worse patient's short-term outcome (higher overall morbidity and mortality rates) but not in worse long-term outcome (overall survival). This may be explained by a more timeconsuming diagnostic workup in patients with a poorer physical status and not by tumor progression.

Acknowledgment The authors are indebted to Mrs. C.M. VollebregtUiterwijk for her dedicated prospective collection of data.

Open Access This article is distributed under the terms of the Creative Commons Attribution Noncommercial License which permits any noncommercial use, distribution, and reproduction in any medium, provided the original author(s) and source are credited.

\section{References}

1. Lerut T, De Leyn P, Coosemans W et al. Surgical strategies in esophageal carcinoma with emphasis on radical lymphadenectomy. Ann Surg 1992;216(5):583-590.

2. Siewert JR, Stein HJ, Feith $M$ et al. Histologic tumor type is an independent prognostic parameter in esophageal cancer: lessons from more than 1,000 consecutive resections at a single center in the Western world. Ann Surg 2001;234(3):360-367. (discussion 368-369).

3. Hulscher JB, van Sandick JW, de Boer AG et al. Extended transthoracic resection compared with limited transhiatal resection for adenocarcinoma of the esophagus. N Engl J Med 2002;347 (21):1662-1669.

4. Eloubeidi MA, Desmond R, Arguedas MR et al. Prognostic factors for the survival of patients with esophageal carcinoma in the U.S.: the importance of tumor length and lymph node status. Cancer 2002;95(7):1434-1443.

5. Wijnhoven BP, Tran KT, Esterman A et al. An evaluation of prognostic factors and tumor staging of resected carcinoma of the esophagus. Ann Surg 2007;245(5):717-725.

6. Richards MA, Westcombe AM, Love SB et al. Influence of delay on survival in patients with breast cancer: a systematic review. Lancet 1999;353(9159):1119-1126. 
7. Ramos M, Esteva M, Cabeza E et al. Relationship of diagnostic and therapeutic delay with survival in colorectal cancer: a review. Eur J Cancer 2007;43(17):2467-2478.

8. Ramos M, Esteva M, Cabeza E et al. Lack of association between diagnostic and therapeutic delay and stage of colorectal cancer. Eur J Cancer 2008;44(4):510-521.

9. Wang J, Liu F, Gao H et al. The symptom-to-treatment delay and stage at the time of treatment in cancer of esophagus. Jpn J Clin Oncol 2008;38(2):87-91.

10. Kotz BS, Croft S, Ferry DR. Do delays between diagnosis and surgery in resectable oesophageal cancer affect survival? A study based on West Midlands cancer registration data. Br J Cancer 2006;95(7):835-840.

11. Rothwell JF, Feehan E, Reid I et al. Delay in treatment for oesophageal cancer. Br J Surg 1997;84(5):690-693.

12. Martin IG, Young S, Sue-Ling H, Johnston D. Delays in the diagnosis of oesophagogastric cancer: a consecutive case series. BMJ 1997;314(7079):467-470.

13. Witzig R, Schonberger B, Fink U et al. Delays in diagnosis and therapy of gastric cancer and esophageal adenocarcinoma. Endoscopy 2006;38(11):1122-1126.

14. Kok TC, Van Lanschot JJB, Siersema PD et al. Neoadjuvant chemotherapy in operable esophageal squamous cell cancer: final report of a phase III multicenter randomized controlled trial. Proc Am Soc Clin Oncol 1997;17:984.
15. van Heijl M, van Lanschot JJ, Koppert LB et al. Neoadjuvant chemoradiation followed by surgery versus surgery alone for patients with adenocarcinoma or squamous cell carcinoma of the esophagus (CROSS). BMC Surg 2008;8:21.

16. Omloo JM, Lagarde SM, Hulscher JB et al. Extended transthoracic resection compared with limited transhiatal resection for adenocarcinoma of the mid/distal esophagus: five-year survival of a randomized clinical trial. Ann Surg 2007;246(6):992-1000. (discussion 1000-1001).

17. Sobin L. TNM classification of malignant tumors, 6th edn. Wiley: New York, 2002.

18. Visser MR, van Lanschot JJ, van der Velden J et al. Quality of life in newly diagnosed cancer patients waiting for surgery is seriously impaired. J Surg Oncol 2006;93(7):571-577.

19. Logan RF, Behan MW, Campion G, Keating NA. Impact of the two-week referral guideline on time to diagnosis and treatment in oesophago-gastric cancer. Clin Med 2003;3(4): 386-387.

20. Spahos T, Hindmarsh A, Cameron E et al. Endoscopy waiting times and impact of the two week wait scheme on diagnosis and outcome of upper gastrointestinal cancer. Postgrad Med J 2005;81 (961):728-730.

21. Rachet B, Maringe C, Nur U et al. Population-based cancer survival trends in England and Wales up to 2007: an assessment of the NHS cancer plan for England. Lancet Oncol 2009;10(4):351369. 